\title{
An Approach of Removing Stroke Based on Touching Pattern for Handwritten Digits Segmentation
}

\author{
Ma Rui ${ }^{1,2}$, Cheng Yaping ${ }^{1,2}$, Gu Yunhua ${ }^{1,2}$ \\ ${ }^{1}$ Jiangsu Engineering Center of Network Monitoring, Nanjing University of Information Science \& Technology, Nanjing, China \\ ${ }^{2}$ Computer and Software Institute, Nanjing University of Information Science \& Technology, Nanjing, China \\ marui_mary@yahoo.com.cn
}

\begin{abstract}
For finding precise segmentation, a new approach to remove and amend stroke connecting touching handwriting digits based on touching pattern is presented. Firstly, background region of touching digits is analyzed by using water reservoirs concept, and then the classification scheme is developed to identify the pattern of touching digits according to the structural features. In single-point touching type, two touching pairs of digits can be connected with a ligature, which can be a component of one of the digits or belong to no digit. After segmentation is performed to get isolated digits. the pattern-oriented ligature analysis investigates whether candidate ligature is a part of digit or a useless stroke and decides to be preserved or be removed. Experimental results show that the proposed approach is effective for segmentation of handwritten touching digits and segmentation errors have been reduced.

Index Terms - Water reservoirs, handwritten digits segmentation, remove stroke
\end{abstract}

\section{Introduction}

Separating handwritten numeral strings has been a popular topic of research for many years, and it plays pivotal role in many numerous applications ${ }^{[1]}$. Separating touching digits is one of the main challenges in handwritten numeral segmentation. Generally numeral strings include isolated digits, touched, overlapped, and noisy or broken digits ${ }^{[2]}$. At present, many approaches have been proposed on segmentation of touching digits ${ }^{[3]}$.

The touching pairs of digits can be connected with a ligature or a part of a digit. The former and latter are considered as ligature and fake ligature, respectively. The ligature is a useless component between two touching digits and it should be removed before recognition because it merely causes recognition error. The fake ligature looks like a ligature, but it is a part of digit and it should be preserved and amended.

In [4] four Drop-fall algorithms are applied at the same time to produce a set of several possible cutting paths. In the following stage, deciding which path is the correct one is involved. This determination is made by using a neural network approach to select the path on the basis of the confidence levels provided by the recognition module. But this method with the interaction between segmentation module and recognition module is complicated, and the result relies on recognition precision strongly.

In this paper, a touching pattern-oriented ligature segmentation processing is proposed. In this strategy classification scheme of touching patterns is developed on the basis of analyzing structural features which are obtained from touching region of connected digits. According to the touching pattern, the segmentation is performed. After segmenting the ligature analysis investigates examine whether the ligature component is a useless component connects two digits which should be removed or a part of digit which should be preserved and amended.

\section{Patterns of Touching Digits}

While writing down digits, it is possible to have adjacent digits connected in several ways which include:

\section{A. Simple touching}

Two digits touch at a single location where there are two possible cases including:

1) Single-segment touching

They share a long continuous stroke segment (see Fig.1(a)).

2) Single-point touching

They are connected with a ligature and share one point. The ligature can either be a component of one of the digits or belong to no digit. The former has an end point of one stroke which touches one side of another stroke, or two end points of two strokes which touch each other. The latter is an extra component with two transition points connecting the digits and it should be removed before recognition. In terms of ligature circumstances, we define four sub-patterns inside single-point touching as below:

a) C_1: The ligature belongs to left digit (see Fig.1(b)).

b) $C_{-} 2$ : The ligature belongs to right digit (see Fig.1(c)).

c) $C_{-} 3$ :The ligature belongs to both left and right digits (see Fig.1(d)).

d) C_4:The ligature is yielded by consecutive writing between digits and belongs to no digit (see Fig.1(e)).

B. Multiple touching

Two digits touch at multiple locations (see Fig.1(f)).

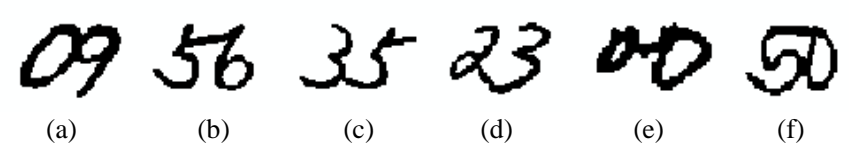

Fig.1 Different patterns of touching digits.

\section{Touching Pattern Indentification}

For performance reasons, to avoid unnecessary 
recognition attempts as much as possible, we propose a pattern-oriented strategy based on the developed classification scheme of touching patterns. We can choose one suitable dropfall method aiming at the pattern of touching digits in order to obtain the best segmentation result.

The problem in this strategy is to find the pattern of touching digits. Considering structural features of touching digits, we can analyze the background region of connected digits to obtain the touching circumstances.

\section{A. Background Analysis.}

Generally, two adjacent digits create large space when they touch each other. In order to describe the large space vividly, we cite water reservoir concept ${ }^{[5]}$ in this paper Water reservoirs can be considered that if water is poured from top or bottom of the numeral, the water will be stored in large space when stopped by the bottom of the numeral external contour. The water stored area can be called reservoir, and the lowermost row of the reservoir can be called baseline (see Fig.2). When water is poured from top or bottom we can obtain the top or bottom reservoirs respectively.

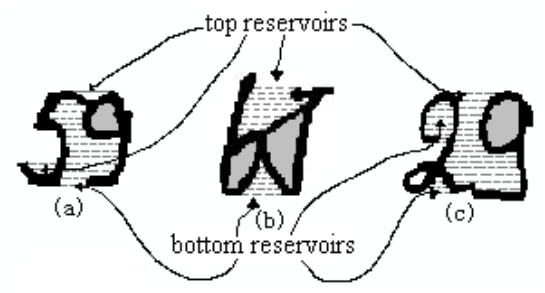

Fig.2 Space created by touching digits.

Our idea is to use water reservoir to obtain the large spaces created by touching digits and analyze the circumstances of junction. The principle is as follows:

Let $\mathrm{CC}$ be a connected component, and its bounding box area is $\mathrm{CW} \times \mathrm{CH}$. The $\mathrm{CC}$ is divided vertically into three areas in proportion with 1: 2: 1 of component width $C W$ and the areas from left to right are denoted as $v_{l}, v_{m}$ and $v_{r}$, respectively. Similarly, the horizontal division of the CC in three areas is done and the corresponding areas from top to bottom are denoted as $h_{t}, h_{m}$ and $h_{b}$. For illustration see Fig.3.

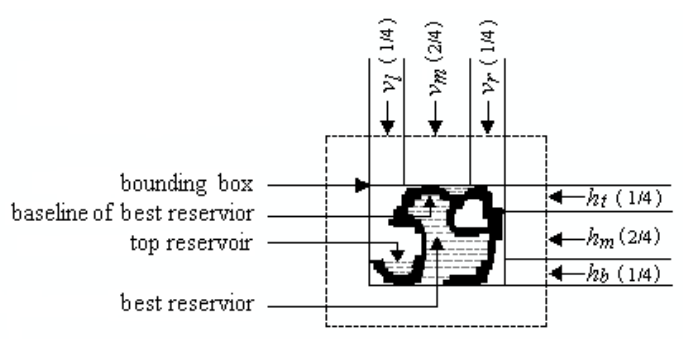

Fig.3 Feature detection approach.

Here, only those reservoirs whose heights are greater than 1/8 of component height $\mathrm{CH}$ and the centers of gravity of reservoirs lie in $\mathrm{vm}$ region are considered for further processing. Meanwhile, the close loops are taken into consideration too. Since handwritten digits may be written in many different ways, the features to be extracted should be independent of individual writing styles. The parameters from topological features are used as follows:

$N W_{U}$ - Number of top reservoirs

$N W_{D}$ - Number of bottom reservoirs

$N W$ - Total number of water reservoirs

$G W_{i}$ - Centre of gravity position of reservoir $W i(1 \leq i \leq N W)$

$B W_{i}$ - Bounding box of reservoir $W i(1 \leq i \leq N W)$

$N L$ - Number of loops

$G L_{k}$ - Centre of gravity position of loop $L_{k}(1 \leq k \leq N L)$

$B L_{k}$ - Bounding box of loop $L_{k}(1 \leq k \leq N L)$

\section{B. Touching Pattern Identification.}

Based on the parameters above, we develop the classification scheme of touching patters represented in pseudo code as follows:
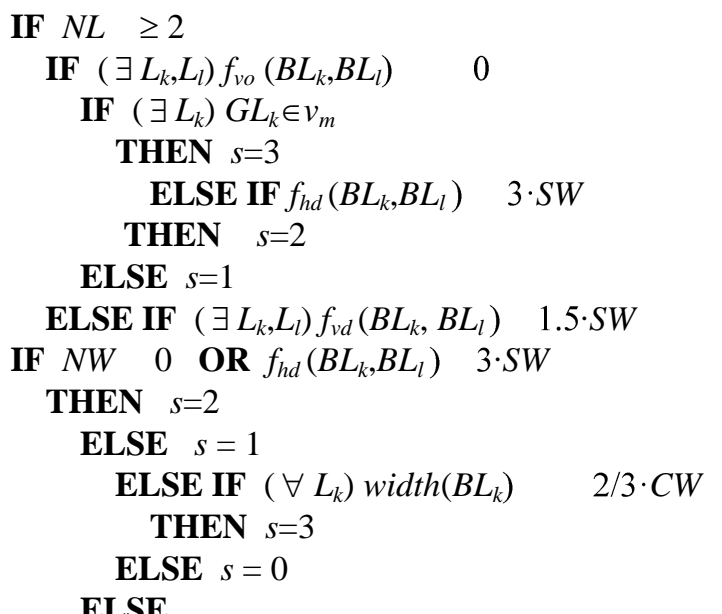

\section{ELSE}

IF $N W \geq 3$

THEN $s=2$

ELSE IF $\left(\exists W_{i}, W_{j}\right) f_{h o}\left(B W_{i}, B W_{j}\right) \quad 0$

THEN $s=2$

ELSE IF $N W_{U}=2$

IF $\left(\exists W_{i}, W_{j}\right) \max \left(w i d t h\left(B W_{i}\right)\right.$, width $\left.\left(B W_{i}\right)\right) \quad 2 / 3 \cdot C W$

THEN $s=2$

ELSE $s=0$

ELSE IF $\left(\exists W_{i}\right)$ height $\left(B W_{i}\right) \geq 3 / 4 \cdot C H \quad \wedge G W_{i} \in v_{m}$ THEN $s=2$

ELSE IF $N L=1$

IF $G L_{k} \in h_{m}$

IF width $\left(B L_{k}\right) \quad 2 / 3 \cdot C W$

IF $N W \quad 0$

THEN $s=3$

ELSE $s=2$

ELSE $s=1$

ELSE

IF $N W \quad 0$

THEN $s=1$

ELSE $s=0$

ELSE $s=0$

where 


$$
s= \begin{cases}0 & \text { isolated } \\ 1 & \text { single-segment touching } \\ 2 & \text { single- point touching } \\ 3 & \text { multiple- segment touching }\end{cases}
$$

$f_{\text {ho }}(A, B)$ - horizontal overlap between rectangle $A$ and $B$

$f_{v o}(A, B)$ - vertical overlap between rectangle $A$ and $B$

$f_{h d}(A, B)$ - horizontal gap between rectangle $A$ and $B$

$f_{v d}(A, B)$ - vertical gap between rectangle $A$ and $B$

\section{Pattern-Oriented removing useless stroke}

In single-point touching type, two touching pairs of digits can be connected with a ligature, which can be a component of one of the digits or belong to no digit. The latter is considered as a useless stroke between two touching digits, and it should be removed after segmentation because it merely causes recognition error. The ligature analysis investigates whether candidate ligature is a part of digit or a useless stroke and decides to be preserved or be removed.

Suppose the left-top point of the image be the coordinate origin. Firstly, the outer contour of the touching digits is separated by the leftmost and the rightmost contour points into upper and lower part denoted by $C_{u p}$ and $C_{d n}$. We describe $U(x), D(x)$ (see Fig.4(a) and (b)) and an approximated measure of vertical width Diff(x) (see Fig.4(c)) are the functions of horizontal coordinate $\mathrm{x}$ bellow: $B_{u p}(x)$ and $B_{d n}(x)$ are maximum and minimum $y$ position according to the image pixels $f(x, y)$ of the $C_{u p}$ and $C_{d n}$ respectively.

$$
\begin{aligned}
& \operatorname{Diff}(\mathrm{x})=D(x)-U(x) \\
& U(x)=\max _{p(x, y)-C u p} y \\
& D(x)=\min _{p(x, y)-C d n} y
\end{aligned}
$$
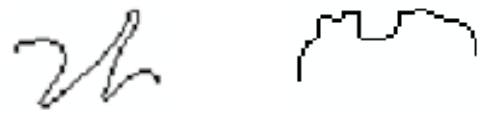

(a) Upper contour
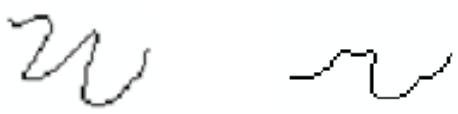

(b) Lower contour

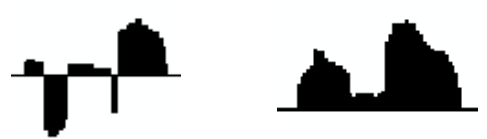

(c) Upper- lower contour difference

Fig.4 Upper- lower contour difference

The both ligatures have two candidate transition points used to detect ligature component, $P_{1}(x, y)$ and $P_{2}(x, y)$ are defined as follows:

$$
\begin{aligned}
& P_{1}(x, y)=\left\{(x, y) \in C_{u p} \mid \text { Diff_(x)/Diff_( }(x+1) \quad T_{1}, \text { Diff_( }(x) \quad 0\right\} \\
& P_{2}(x, y)=\left\{(x, y) \in C_{d n} \mid \operatorname{Diff} \_(x+1) / D i f f \_(x) \quad T_{2}, \text { Diff_( }(x) \quad 0\right\}
\end{aligned}
$$

The ligature analysis is to examine whether the ligature component is a useless component connects two digits or a part of digit. In case of ligature, $P_{1}(x, y)$ and $P_{2}(x, y)$ are starting and ending points which are appeared in sequence order. But in case of fake ligature, the order of $P_{1}(x, y)$ and $P_{2}(x, y)$ are opposite. The analysis is processed as follows:

1) From segment position $x_{0}$ right break position is detected rightward until the point is encountered which satisfies one of the above conditions, denoted by $X_{\text {right }}$. Similarly, the left break point $X_{\text {left }}$ is abtained.

2) The ligature component is considered as a real ligature when the conditions listed below are satisfied.

a) Starting point $P_{1}(x, y)$ and ending point $P_{2}(x, y)$ are appeared in sequence order, i.e. $x_{1}<x_{2}, x_{1}$ and $x_{2}$ are $x$ coordinates of $P_{1}(x, y)$ and $P_{2}(x, y)$ respectively. $\left.\left.y_{\text {middle }}\right)\right\}$

b) $\left.\left.\left\{\left(x_{1}, y_{1}^{\prime}\right)\right\} \in C_{d n} \wedge\left(x_{2}, y_{2}^{\prime}\right) \in C_{d n}\right]\right\} \wedge\left\{\left(y_{1}^{\prime}, y_{\text {middle }}\right) \wedge\left(y_{2}^{\prime}\right.\right.$

Herein, $y_{1}^{\prime}$ and $y_{2}^{\prime}$ are $y$-coordinates of $C_{d n}\left(x_{1}, y_{1}^{\prime}\right)$ and $C d n\left(x_{2}, y_{2}^{\prime}\right)$ respectively, and $y_{\text {middle }}$ is the middle height line.

c) If average value of vertical difference between starting and ending break points of candidate ligature is smaller than $T_{d i f f}$, then the candidate ligature is considered as ligature.

$$
\frac{1}{x_{2}-x_{1}+1} \sum_{x=x_{1}}^{x_{2}} D i f f(x)<T
$$

$T_{\text {diff }}$ is calculated by averaging $D_{v}$ and $T$ is calculated from the average vertical boundary difference $D(x)$.

3 ) If any one of the above conditions is not satisfied, the ligature component is considered as a part of digit and there is need for amending the boundary box of the segmented digit.

For $C_{-} 1$ and $C_{-} 3$ touching pattern, taking $X_{\text {left }}=X_{\text {right }}$ $\left(X_{\text {right }}-X_{\text {leff }}^{\prime}\right) / 3$ as the right boundary position of the left digit.

For $C \_2$ and $C_{-} 3$ touching pattern,taking $X_{\text {right }}=X_{\text {left }}^{\prime}+$ $\left(X_{\text {right }}-X_{\text {left }}^{\prime}\right) / 3$ as the left boundary position of the right digit.

4) Otherwise, a candidate ligature component is considered as a useless component connects two digits, and there is need to removed it. Let $X_{\text {left }}=X_{\text {left }}$, and remove the left connected position on the segment between $\left(X_{\text {left }}, U\left(X_{\text {left }}\right)\right)$ and $\left(X_{\text {left }}, D\left(X_{\text {left }}\right)\right)$. Similarly, remove the right connected position on the segment between $\left(X_{\text {right }}, U\left(X_{\text {right }}\right)\right)$ and $\left(X_{\text {right }}, D\left(X_{\text {right }}\right)\right)$.

In Fig.5, above process is presented. The ligature component connecting " 2 " and " 6 " is a part of digit " 2 ", and should be reserved and amended. The ligature component connecting " 0 " and " 0 " is an extra part of two digits " 0 ", and should be removed. 


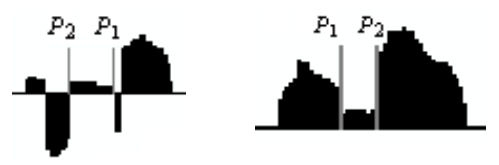

(a) Detecting transition points

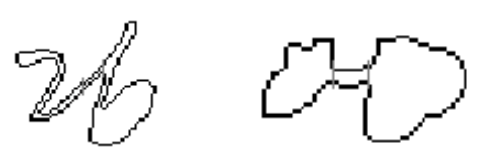

(b) Verifying the left and the right segmentation points

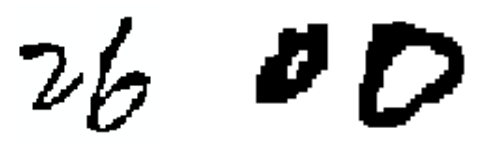

(c) Removing useless strokes

Fig.5 Process of removing useless strokes

\section{V . Experiments}

To evaluate the proposed strategy for getting more precise results of segmentation, we have carried out experiments with NJUST603HW database. The database contains 623 binary images of handwritten courtesy amount string which are taken from the bank checks currently used in China. From all the images we extract 3783 sub-string images of isolated digits and 1591 sub-string images of touching digits which includes 911 images with single-point touching, 412 images with single-segment touching and 268 images with multiple-touching.

The performance of the classification scheme is run with 5374 total sub-string images and the result is shown in TABLE I. The developed scheme has $98.29 \%$ accuracy for classifying touching digits patterns.

The pattern-oriented segmentation and removing stroke performance is conducted on 1557 connected digits which touching patterns have been classified correctly and the result is listed in TABLE II. With the manual verification of the segmentation, $96.66 \%$ of digits were successively segmented.

TABLE I Result of Our Classification Scheme

\begin{tabular}{|r|c|c|c|}
\hline & \# Images & \# Accuracy & Accuracy (\%) \\
\hline Isolated digits & 3783 & 3725 & 98.47 \\
\hline Touching digits & 1591 & 1557 & 97.86 \\
\hline Total & 5374 & 5282 & 98.29 \\
\hline
\end{tabular}

TABLE II Result of Pattern-Oriented Strategy for Segmentation

\begin{tabular}{|c|c|c|}
\hline Touching pattern & \# Touching digits & $\begin{array}{c}\text { Segmentation accuracy } \\
(\%)\end{array}$ \\
\hline $\begin{array}{c}\text { Single point } \\
\text { touching }\end{array}$ & 891 & 97.08 \\
\hline $\begin{array}{c}\text { Single segment } \\
\text { touching }\end{array}$ & 406 & 96.31 \\
\hline Multiple touching & 260 & 95.78 \\
\hline Total & 1557 & 96.66 \\
\hline
\end{tabular}

TABLE III Result of our pattern-oriented segmentation

\begin{tabular}{|c|c|c|c|}
\hline $\begin{array}{c}\text { \# Touching } \\
\text { digits }\end{array}$ & Algorithm & $\begin{array}{c}\text { Segmentation } \\
\text { accuracy (\%) }\end{array}$ & $\begin{array}{c}\text { Recognition } \\
\text { accuracy (\%) }\end{array}$ \\
\hline \multirow{3}{*}{1591} & DD & 92.28 & 86.43 \\
\cline { 2 - 4 } & FA & 93.37 & 86.77 \\
\cline { 2 - 4 } & DF & 89.94 & 81.65 \\
\cline { 2 - 4 } & Ours & 95.60 & 88.18 \\
\hline
\end{tabular}

Besides, TABLE III gives the performance comparison between ours and several successive algorithms described in other literatures: traditional Drop-fall algorithm (DF) [6], algorithm of distance difference from upper contour to lower contour (DD) [7] and foreground analysis algorithm (FA) [8]. By using our strategy $95.6 \%$ of touching digits were correctly segmented exceeding the other algorithms, and recognition accuracy is increased consequently. From Table 3, we can see that our algorithm works better than others.

\section{VI . Conclusion}

In the strategy background region of touching digits is analyzed in order to identify the touching pattern. After separating touching digits, useless stroke is removed according to the touching pattern to get more precise results. The advantage of this strategy is the segmentation procedure can be implemented conveniently.

\section{Acknowledgment}

This paper was supported by Computer and Software Institute, Nanjing University of Information Science \& Technology Foundation 20100340.

\section{References}

[1] Bul O., Polat F. and Alhajj R., "Multi-Agent Reinforcement Learning Using Function Approximation,” IEEE-SMC,2000,Vol.30, No.4, pp.485497.

[2] K.K. Kim, J.H. Kim, and C.Y. Suen, "Segmentation-Based Recognition of Handwritten Touching Pairs of Digits Using Structural Features," Pattern Recognition Letters, 2002, Vol. 23, pp.13-24.

[3] Chen Y.K., Wang J.F., "Segmentation of single- or multiple-touching handwritten numeral string using background and foreground analysis," IEEE Transaction on Pattern Analysis and Machine Intelligence, 2000, pp.1304-1317.

[4] F. Kimura and M. Shridhar, "Segmentation-recognition algorithm for zip code field recognition,' Mach. Vision Appl, Vol.5,No.3, pp.199-210, 1992.

[5] U. Pal, A. Belaid, and Ch. Choisy, "Touching Numeral Segmentation Using Water Reservoir Concept," Pattern Recognition Letters, 2003, Vol. 24, pp. 261-272.

[6] Congedo G., Dimauro G., and Impedovo S., "Segmentation of numeric strings," Proceeding of the Third International Conference on Document Analysis and Recognition, 14-16 Aug 1995, pp.1038-1041.

[7] Fujisawa H, Nakano Y, and Kurino K, "Segmentation methods for character recognition: from segmentation to document structure analysis," Proceedings of the IEEE, 1992, Vol.80, No. 7, pp.1079-1092

[8] Oliveira L S, "A new approach to segment handwritten digits," Seventh International Workshop on Frontiers in Handwritten Recognition (IWFHR-7), 2000,pp.578-582. 